Acta Crystallographica Section E

Structure Reports

Online

ISSN 1600-5368

\section{4-Isopropyl-N-phenylcyclohexa-1,3- diene-1-carboxamide}

\section{Yan-qing Gao, Shi-bin Shang,* Jian Li, Xu Xu and Xiao-ping Rao}

Institute of Chemical Industry of Forest Products, Chinese Academy of Forestry, Nanjing 210042, People's Republic of China

Correspondence e-mail: shangsb@hotmail.com

Received 24 August 2010; accepted 30 August 2010

Key indicators: single-crystal X-ray study; $T=293 \mathrm{~K}$; mean $\sigma(\mathrm{C}-\mathrm{C})=0.003 \AA$; $R$ factor $=0.056 ; w R$ factor $=0.175 ;$ data-to-parameter ratio $=15.2$.

In the crystal structure of the title compound, $\mathrm{C}_{16} \mathrm{H}_{19} \mathrm{NO}$, molecules are linked through a pair of $\mathrm{N}-\mathrm{H} \cdots \mathrm{O}$ hydrogen bonds, forming chains along the $a$ axis.

\section{Related literature}

The title compound was obtained by reaction of dihydrocumic acid, obtained from nopinic acid through dehydration, and aniline. For the preparation and structure of nopinic acid, see: Ma et al. (2007); Gao et al. (2009). For the preparation of dihydrocumic acid, see: Jin \& Ha (2006) For oxidation of $\beta$ pinene, see: Winstein \& Holness (1955).<smiles>CC(C)C1=CC=C(C(=O)Nc2ccccc2)CC1</smiles>

\section{Experimental}

Crystal data

$\mathrm{C}_{16} \mathrm{H}_{19} \mathrm{NO}$

$M_{r}=241.32$
Triclinic, $P \overline{1}$

$a=5.226(1) \AA$

$b=9.783(2) \AA$

$c=13.810(3) \AA$

$\alpha=88.31(3)^{\circ}$

$\beta=88.01(3)^{\circ}$

$\gamma=76.13(2)^{\circ}$

$V=684.9(2) \AA^{3}$

$Z=2$

Mo $K \alpha$ radiation

$\mu=0.07 \mathrm{~mm}^{-1}$

$T=293 \mathrm{~K}$

$0.30 \times 0.20 \times 0.20 \mathrm{~mm}$

\section{Data collection}

Enraf-Nonius CAD-4 diffractometer

Absorption correction: $\psi$ scan (North et al., 1968)

$T_{\min }=0.979, T_{\max }=0.986$

2789 measured reflections

\author{
2491 independent reflections \\ 1901 reflections with $I>2 \sigma(I)$ \\ $R_{\text {int }}=0.013$ \\ 3 standard reflections every 200 \\ reflections \\ intensity decay: $1 \%$
}

Refinement

$R\left[F^{2}>2 \sigma\left(F^{2}\right)\right]=0.056$

$w R\left(F^{2}\right)=0.175$

$S=1.01$

2491 reflections

164 parameters

$\mathrm{H}$-atom parameters constrained

$\Delta \rho_{\max }=0.24{\mathrm{e} \AA^{-3}}^{-3}$

$\Delta \rho_{\min }=-0.22{\mathrm{e} \AA^{-3}}^{-3}$

Table 1

Hydrogen-bond geometry $\left(\AA{ }^{\circ}\right)$.

\begin{tabular}{lllll}
\hline$D-\mathrm{H} \cdots A$ & $D-\mathrm{H}$ & $\mathrm{H} \cdots A$ & $D \cdots A$ & $D-\mathrm{H} \cdots A$ \\
\hline $\mathrm{N}-\mathrm{H} 0 A \cdots \mathrm{O}^{\mathrm{i}}$ & 0.86 & 2.27 & $3.054(2)$ & 151 \\
\hline
\end{tabular}

Symmetry code: (i) $x+1, y, z$.

Data collection: CAD-4 EXPRESS (Enraf-Nonius, 1994); cell refinement: CAD-4 EXPRESS; data reduction: XCAD4 (Harms \& Wocadlo, 1995); program(s) used to solve structure: SHELXS97 (Sheldrick, 2008); program(s) used to refine structure: SHELXL97 (Sheldrick, 2008); molecular graphics: SHELXTL (Sheldrick, 2008); software used to prepare material for publication: SHELXL97.

Supplementary data and figures for this paper are available from the IUCr electronic archives (Reference: DS2053).

\section{References}

Enraf-Nonius (1994). CAD-4 EXPRESS. Enraf-Nonius, Delft, The Netherlands.

Gao, Y.-Q., Shang, S.-B., Xu, X., Rao, X.-P. \& Wang, H.-X. (2009). Acta Cryst. E65, o2748.

Harms, K. \& Wocadlo, S. (1995). XCAD4. University of Marburg, Germany. Jin, J. Z. \& Ha, C. Y. (2006). Chem. Ind. For. Prod. 26, 27-30.

Ma, S. Y., Shen, M. M. \& Ha, C. Y. (2007). Chem. Ind. For. Prod. 27, 114-116.

North, A. C. T., Phillips, D. C. \& Mathews, F. S. (1968). Acta Cryst. A24, 351359.

Sheldrick, G. M. (2008). Acta Cryst. A64, 112-122.

Winstein, S. \& Holness, N. J. (1955). J. Am. Chem. Soc. 77, 3054-3061. 


\section{supporting information}

Acta Cryst. (2010). E66, o2490 [doi:10.1107/S1600536810034859]

\section{4-Isopropyl-N-phenylcyclohexa-1,3-diene-1-carboxamide}

\section{Yan-qing Gao, Shi-bin Shang, Jian Li, Xu Xu and Xiao-ping Rao}

\section{S1. Comment}

Nopinic acid is an important material prepared by oxidation of beta-pinene (Ma, 2007), and the crystal structure of nopinic acid has been reported (Gao,2009). From nopinic acid, dihydrocumic acid was obtained through dehydration. The title compound was got by reaction of dihydrocumic acid and aniline. In this work, we describe the crystal structure of the title compound. The asymmetric unit consists of one crystallographically independent molecule. The independent molecules are linked through a pair of $\mathrm{N}-\mathrm{H} \cdots \mathrm{O}$ hydrogen bonds forming a polymer.

The molecular structure is shown in Fig. 1 and the crystal packing in Fig. 2, where the dash line indicates $\mathrm{N}-\mathrm{H} \cdots \mathrm{O}$ hydrogen bonds. The bond lengths and angles are given in Table 1.

\section{S2. Experimental}

Dihydrocumic acid was $(5.0 \mathrm{~g})$ was dissolved in dichlomethane $(100 \mathrm{ml})$ while stirring vigorously, thionyl chloride $(6.6$ $\mathrm{ml}$ ) was dropped. The reaction was maintained during $6 \mathrm{~h}$ at the temperature of reflux. After removing dichlomethane and redundant thionyl chloride, the carboxylic acid chloride was obtained, which was then dropped in a mixture of dichlomethane $(100 \mathrm{ml})$, triethylamine $(6.1 \mathrm{ml})$ and aniline $(5.6 \mathrm{~g})$. The reaction was stayed over at room temperature. After reagent was romoved, the crude product was crystallized with ethanol, then the title conpound was gained. Crystals of the title compound suitable for X-ray diffraction were obtained by slow evaporation of a solution of ethanol. The crystal data were collected on an Enraf-Nonius CAD-4 difractometer. Data collection and cell refinement were performed using Enraf-Nonius CAD-4 Software.

\section{S3. Refinement}

All $\mathrm{H}$ atoms bonded to the $\mathrm{C}$ atoms were placed geometrically at the distances of $0.96-0.98 \AA$ and included in the refinement in riding motion approximation with $U_{\text {iso }}(\mathrm{H})=1.2$ or $1.5 U_{\text {eq }}$ of the carrier atom. $\mathrm{H}$ atoms bonded to the $\mathrm{N}$ atoms were fixed. 


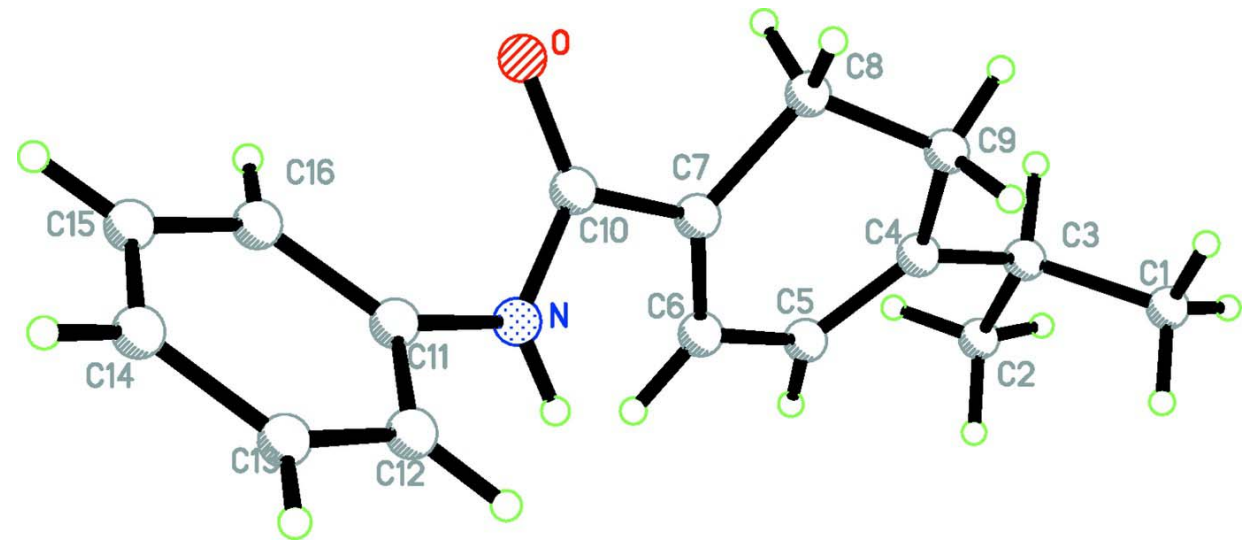

\section{Figure 1}

A view of the molecular structure of (I), showing displacement ellipsoids at the $30 \%$ probability level. 


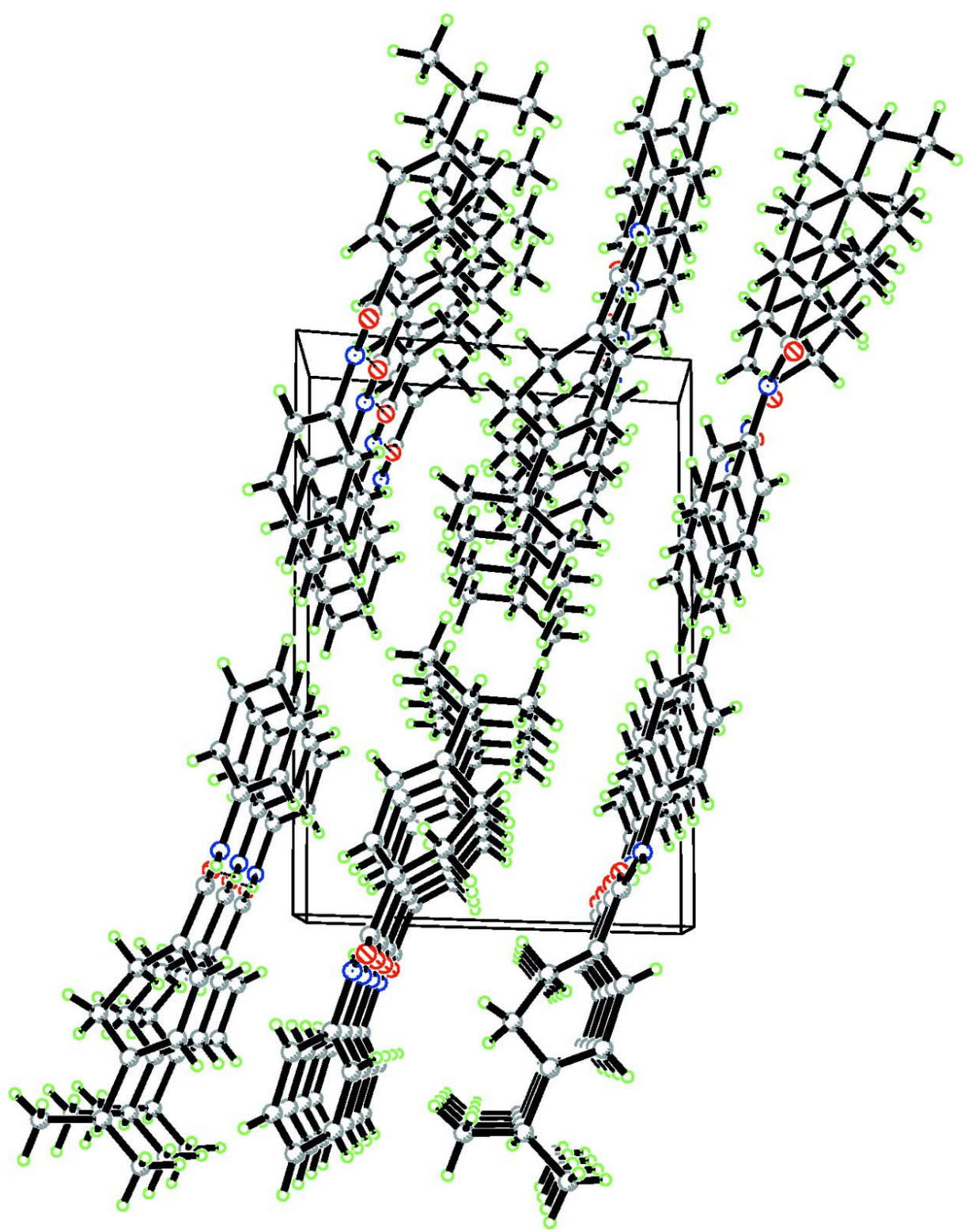

Figure 2

A view of the packing of the title compound.

4-Isopropyl-N-phenylcyclohexa-1,3-diene-1-carboxamide

Crystal data

$\mathrm{C}_{16} \mathrm{H}_{19} \mathrm{NO}$

$M_{r}=241.32$

Triclinic, $P \overline{1}$

Hall symbol: -P 1

$a=5.226(1) \AA$

$b=9.783(2) \AA$

$c=13.810(3) \AA$

$\alpha=88.31(3)^{\circ}$

$\beta=88.01(3)^{\circ}$

$$
\begin{aligned}
& \gamma=76.13(2)^{\circ} \\
& V=684.9(2) \AA^{3} \\
& Z=2 \\
& F(000)=260 \\
& D_{\mathrm{x}}=1.170 \mathrm{Mg} \mathrm{m}{ }^{-3} \\
& \text { Mo } K \alpha \text { radiation, } \lambda=0.71073 \AA \\
& \text { Cell parameters from } 25 \text { reflections } \\
& \theta=9-13^{\circ} \\
& \mu=0.07 \mathrm{~mm}^{-1}
\end{aligned}
$$


$T=293 \mathrm{~K}$

Rod, colourless

Data collection

Enraf-Nonius CAD-4 diffractometer

Radiation source: fine-focus sealed tube

Graphite monochromator

$\omega / 2 \theta$ scans

Absorption correction: $\psi$ scan

(North et al., 1968)

$T_{\min }=0.979, T_{\max }=0.986$

2789 measured reflections

\section{Refinement}

Refinement on $F^{2}$

Least-squares matrix: full

$R\left[F^{2}>2 \sigma\left(F^{2}\right)\right]=0.056$

$w R\left(F^{2}\right)=0.175$

$S=1.01$

2491 reflections

164 parameters

0 restraints

Primary atom site location: structure-invariant direct methods

Secondary atom site location: difference Fourier map
$0.30 \times 0.20 \times 0.20 \mathrm{~mm}$

2491 independent reflections

1901 reflections with $I>2 \sigma(I)$

$R_{\text {int }}=0.013$

$\theta_{\max }=25.3^{\circ}, \theta_{\min }=1.5^{\circ}$

$h=0 \rightarrow 6$

$k=-11 \rightarrow 11$

$l=-16 \rightarrow 16$

3 standard reflections every 200 reflections

intensity decay: $1 \%$

Hydrogen site location: inferred from

neighbouring sites

$\mathrm{H}$-atom parameters constrained

$w=1 /\left[\sigma^{2}\left(F_{\mathrm{o}}^{2}\right)+(0.1 P)^{2}+0.190 P\right]$

where $P=\left(F_{\mathrm{o}}^{2}+2 F_{\mathrm{c}}^{2}\right) / 3$

$(\Delta / \sigma)_{\max }<0.001$

$\Delta \rho_{\max }=0.24$ e $\AA^{-3}$

$\Delta \rho_{\min }=-0.22$ e $\AA^{-3}$

Extinction correction: SHELXL97 (Sheldrick, 2008), $\mathrm{Fc}^{*}=\mathrm{kFc}\left[1+0.001 \mathrm{xFc}^{2} \lambda^{3} / \sin (2 \theta)\right]^{-1 / 4}$

Extinction coefficient: 0.086 (14)

\section{Special details}

Geometry. All e.s.d.'s (except the e.s.d. in the dihedral angle between two 1.s. planes) are estimated using the full covariance matrix. The cell e.s.d.'s are taken into account individually in the estimation of e.s.d.'s in distances, angles and torsion angles; correlations between e.s.d.'s in cell parameters are only used when they are defined by crystal symmetry. An approximate (isotropic) treatment of cell e.s.d.'s is used for estimating e.s.d.'s involving 1.s. planes.

Refinement. Refinement of $F^{2}$ against ALL reflections. The weighted $R$-factor $w R$ and goodness of fit $S$ are based on $F^{2}$, conventional $R$-factors $R$ are based on $F$, with $F$ set to zero for negative $F^{2}$. The threshold expression of $F^{2}>\sigma\left(F^{2}\right)$ is used only for calculating $R$-factors (gt) etc. and is not relevant to the choice of reflections for refinement. $R$-factors based on $F^{2}$ are statistically about twice as large as those based on $F$, and $R$ - factors based on ALL data will be even larger.

Fractional atomic coordinates and isotropic or equivalent isotropic displacement parameters $\left(\hat{A}^{2}\right)$

\begin{tabular}{lllll}
\hline & $x$ & $y$ & $z$ & $U_{\text {iso }} / U_{\text {eq }}$ \\
\hline $\mathrm{N}$ & $0.2449(3)$ & $0.16425(18)$ & $0.09778(11)$ & $0.0458(4)$ \\
$\mathrm{H} 0 \mathrm{~A}$ & 0.3953 & 0.1648 & 0.0706 & $0.055^{*}$ \\
$\mathrm{O}$ & $-0.1955(3)$ & $0.2062(2)$ & $0.07382(11)$ & $0.0737(6)$ \\
$\mathrm{C} 1$ & $0.3023(9)$ & $0.3380(4)$ & $-0.4181(2)$ & $0.1092(13)$ \\
$\mathrm{H} 1 \mathrm{~A}$ & 0.2422 & 0.2525 & -0.4160 & $0.164^{*}$ \\
$\mathrm{H} 1 \mathrm{~B}$ & 0.4855 & 0.3171 & -0.4026 & $0.164^{*}$ \\
$\mathrm{H} 1 \mathrm{C}$ & 0.2796 & 0.3800 & -0.4818 & $0.164^{*}$ \\
$\mathrm{C} 2$ & $0.2353(6)$ & $0.5737(3)$ & $-0.3466(2)$ & $0.0852(9)$ \\
$\mathrm{H} 2 \mathrm{~A}$ & 0.2377 & 0.6100 & -0.4119 & $0.128^{*}$ \\
$\mathrm{H} 2 \mathrm{~B}$ & 0.4093 & 0.5560 & -0.3213 & $0.128^{*}$ \\
$\mathrm{H} 2 \mathrm{C}$ & 0.1162 & 0.6414 & -0.3072 & $0.128^{*}$ \\
$\mathrm{C} 3$ & $0.1450(5)$ & $0.4384(3)$ & $-0.34559(17)$ & $0.0661(7)$
\end{tabular}




$\begin{array}{lllll}\text { H3A } & -0.0370 & 0.4629 & -0.3675 & 0.079^{*} \\ \text { C4 } & 0.1360(4) & 0.3777(2) & -0.24416(15) & 0.0524(6) \\ \text { C5 } & 0.2943(5) & 0.2582(2) & -0.21217(15) & 0.0574(6) \\ \text { H5A } & 0.4178 & 0.2047 & -0.2547 & 0.069^{*} \\ \text { C6 } & 0.2776(4) & 0.2095(2) & -0.11182(15) & 0.0524(5) \\ \text { H6A } & 0.4138 & 0.1391 & -0.0872 & 0.063^{*} \\ \text { C7 } & 0.0686(4) & 0.2651(2) & -0.05479(14) & 0.0459(5) \\ \text { C8 } & -0.1475(5) & 0.3782(3) & -0.09523(18) & 0.0720(8) \\ \text { H8A } & -0.2305 & 0.4400 & -0.0436 & 0.086^{*} \\ \text { H8B } & -0.2800 & 0.3359 & -0.1211 & 0.086^{*} \\ \text { C9 } & -0.0495(6) & 0.4630(3) & -0.17347(19) & 0.0803(9) \\ \text { H9A } & -0.1993 & 0.5186 & -0.2078 & 0.096^{*} \\ \text { H9B } & 0.0375 & 0.5277 & -0.1439 & 0.096^{*} \\ \text { C10 } & 0.0261(4) & 0.2093(2) & 0.04320(14) & 0.0479(5) \\ \text { C11 } & 0.2431(3) & 0.1169(2) & 0.19526(13) & 0.0421(5) \\ \text { C12 } & 0.4082(4) & 0.1589(2) & 0.25855(15) & 0.0504(5) \\ \text { H12A } & 0.5193 & 0.2149 & 0.2360 & 0.061^{*} \\ \text { C13 } & 0.4081(5) & 0.1179(3) & 0.35443(16) & 0.0610(6) \\ \text { H13A } & 0.5183 & 0.1471 & 0.3966 & 0.073^{*} \\ \text { C14 } & 0.2471(5) & 0.0344(3) & 0.38850(17) & 0.0670(7) \\ \text { H14A } & 0.2448 & 0.0082 & 0.4538 & 0.080^{*} \\ \text { C15 } & 0.0880(5) & -0.0104(3) & 0.32473(19) & 0.0678(7) \\ \text { H15A } & -0.0184 & -0.0691 & 0.3471 & 0.081^{*} \\ \text { C16 } & 0.0850(4) & 0.0304(2) & 0.22861(16) & 0.0552(6) \\ \text { H16A } & -0.0233 & -0.0001 & 0.1863 & 0.066^{*}\end{array}$

Atomic displacement parameters $\left(\AA^{2}\right)$

\begin{tabular}{lllllll}
\hline & $U^{11}$ & $U^{22}$ & $U^{33}$ & $U^{12}$ & $U^{13}$ & $U^{23}$ \\
\hline $\mathrm{N}$ & $0.0335(8)$ & $0.0635(11)$ & $0.0401(9)$ & $-0.0120(7)$ & $-0.0014(7)$ & $0.0048(7)$ \\
$\mathrm{O}$ & $0.0374(8)$ & $0.1279(16)$ & $0.0576(10)$ & $-0.0258(9)$ & $-0.0067(7)$ & $0.0244(10)$ \\
$\mathrm{C} 1$ & $0.194(4)$ & $0.089(2)$ & $0.0460(15)$ & $-0.040(2)$ & $0.0213(19)$ & $0.0009(14)$ \\
$\mathrm{C} 2$ & $0.098(2)$ & $0.0749(18)$ & $0.086(2)$ & $-0.0294(16)$ & $0.0137(16)$ & $0.0103(15)$ \\
$\mathrm{C} 3$ & $0.0751(16)$ & $0.0754(16)$ & $0.0517(13)$ & $-0.0268(13)$ & $-0.0054(11)$ & $0.0128(12)$ \\
$\mathrm{C} 4$ & $0.0595(13)$ & $0.0566(13)$ & $0.0453(12)$ & $-0.0216(11)$ & $-0.0079(9)$ & $0.0018(9)$ \\
$\mathrm{C} 5$ & $0.0653(14)$ & $0.0595(13)$ & $0.0444(12)$ & $-0.0104(11)$ & $0.0073(10)$ & $-0.0029(10)$ \\
$\mathrm{C} 6$ & $0.0548(12)$ & $0.0554(12)$ & $0.0449(11)$ & $-0.0095(10)$ & $-0.0012(9)$ & $0.0025(9)$ \\
$\mathrm{C} 7$ & $0.0401(10)$ & $0.0592(12)$ & $0.0409(11)$ & $-0.0160(9)$ & $-0.0077(8)$ & $0.0010(9)$ \\
$\mathrm{C} 8$ & $0.0486(13)$ & $0.101(2)$ & $0.0571(14)$ & $-0.0021(13)$ & $-0.0023(10)$ & $0.0125(13)$ \\
$\mathrm{C} 9$ & $0.0794(18)$ & $0.0831(19)$ & $0.0611(15)$ & $0.0122(15)$ & $0.0004(13)$ & $0.0152(13)$ \\
$\mathrm{C} 10$ & $0.0378(11)$ & $0.0647(13)$ & $0.0420(11)$ & $-0.0137(9)$ & $-0.0035(8)$ & $0.0012(9)$ \\
$\mathrm{C} 11$ & $0.0331(9)$ & $0.0500(11)$ & $0.0400(10)$ & $-0.0036(8)$ & $-0.0006(7)$ & $0.0009(8)$ \\
$\mathrm{C} 12$ & $0.0420(11)$ & $0.0614(13)$ & $0.0483(12)$ & $-0.0132(9)$ & $-0.0048(9)$ & $0.0024(10)$ \\
$\mathrm{C} 13$ & $0.0540(13)$ & $0.0807(16)$ & $0.0450(12)$ & $-0.0084(12)$ & $-0.0103(10)$ & $-0.0001(11)$ \\
$\mathrm{C} 14$ & $0.0555(14)$ & $0.0907(18)$ & $0.0456(12)$ & $-0.0021(13)$ & $0.0013(10)$ & $0.0171(12)$ \\
$\mathrm{C} 15$ & $0.0517(13)$ & $0.0821(17)$ & $0.0687(16)$ & $-0.0180(12)$ & $0.0009(11)$ & $0.0259(13)$ \\
$\mathrm{C} 16$ & $0.0451(11)$ & $0.0649(14)$ & $0.0581(13)$ & $-0.0180(10)$ & $-0.0079(9)$ & $0.0090(11)$ \\
& & & & & & \\
\hline
\end{tabular}


Geometric parameters $\left(\AA,{ }^{\circ}\right)$

\begin{tabular}{|c|c|c|c|}
\hline $\mathrm{N}-\mathrm{C} 10$ & $1.367(2)$ & C6-H6A & 0.9300 \\
\hline $\mathrm{N}-\mathrm{C} 11$ & $1.411(2)$ & $\mathrm{C} 7-\mathrm{C} 10$ & $1.474(3)$ \\
\hline $\mathrm{N}-\mathrm{H} 0 \mathrm{~A}$ & 0.8600 & $\mathrm{C} 7-\mathrm{C} 8$ & $1.490(3)$ \\
\hline $\mathrm{O}-\mathrm{C} 10$ & $1.226(2)$ & $\mathrm{C} 8-\mathrm{C} 9$ & $1.494(4)$ \\
\hline $\mathrm{C} 1-\mathrm{C} 3$ & $1.502(4)$ & $\mathrm{C} 8-\mathrm{H} 8 \mathrm{~A}$ & 0.9700 \\
\hline $\mathrm{C} 1-\mathrm{H} 1 \mathrm{~A}$ & 0.9600 & $\mathrm{C} 8-\mathrm{H} 8 \mathrm{~B}$ & 0.9700 \\
\hline $\mathrm{C} 1-\mathrm{H} 1 \mathrm{~B}$ & 0.9600 & C9-H9A & 0.9700 \\
\hline $\mathrm{C} 1-\mathrm{H} 1 \mathrm{C}$ & 0.9600 & C9-H9B & 0.9700 \\
\hline $\mathrm{C} 2-\mathrm{C} 3$ & $1.507(4)$ & $\mathrm{C} 11-\mathrm{C} 16$ & $1.377(3)$ \\
\hline $\mathrm{C} 2-\mathrm{H} 2 \mathrm{~A}$ & 0.9600 & $\mathrm{C} 11-\mathrm{C} 12$ & $1.386(3)$ \\
\hline $\mathrm{C} 2-\mathrm{H} 2 \mathrm{~B}$ & 0.9600 & $\mathrm{C} 12-\mathrm{C} 13$ & $1.372(3)$ \\
\hline $\mathrm{C} 2-\mathrm{H} 2 \mathrm{C}$ & 0.9600 & $\mathrm{C} 12-\mathrm{H} 12 \mathrm{~A}$ & 0.9300 \\
\hline $\mathrm{C} 3-\mathrm{C} 4$ & $1.509(3)$ & $\mathrm{C} 13-\mathrm{C} 14$ & $1.370(4)$ \\
\hline $\mathrm{C} 3-\mathrm{H} 3 \mathrm{~A}$ & 0.9800 & $\mathrm{C} 13-\mathrm{H} 13 \mathrm{~A}$ & 0.9300 \\
\hline $\mathrm{C} 4-\mathrm{C} 5$ & $1.333(3)$ & $\mathrm{C} 14-\mathrm{C} 15$ & $1.381(4)$ \\
\hline $\mathrm{C} 4-\mathrm{C} 9$ & $1.478(4)$ & $\mathrm{C} 14-\mathrm{H} 14 \mathrm{~A}$ & 0.9300 \\
\hline $\mathrm{C} 5-\mathrm{C} 6$ & $1.458(3)$ & $\mathrm{C} 15-\mathrm{C} 16$ & $1.374(3)$ \\
\hline $\mathrm{C} 5-\mathrm{H} 5 \mathrm{~A}$ & 0.9300 & $\mathrm{C} 15-\mathrm{H} 15 \mathrm{~A}$ & 0.9300 \\
\hline $\mathrm{C} 6-\mathrm{C} 7$ & $1.338(3)$ & $\mathrm{C} 16-\mathrm{H} 16 \mathrm{~A}$ & 0.9300 \\
\hline $\mathrm{C} 10-\mathrm{N}-\mathrm{C} 11$ & $125.04(16)$ & $\mathrm{C} 7-\mathrm{C} 8-\mathrm{C} 9$ & 112.09 (19) \\
\hline $\mathrm{C} 10-\mathrm{N}-\mathrm{H} 0 \mathrm{~A}$ & 117.5 & $\mathrm{C} 7-\mathrm{C} 8-\mathrm{H} 8 \mathrm{~A}$ & 109.2 \\
\hline $\mathrm{C} 11-\mathrm{N}-\mathrm{H} 0 \mathrm{~A}$ & 117.5 & $\mathrm{C} 9-\mathrm{C} 8-\mathrm{H} 8 \mathrm{~A}$ & 109.2 \\
\hline $\mathrm{C} 3-\mathrm{C} 1-\mathrm{H} 1 \mathrm{~A}$ & 109.5 & $\mathrm{C} 7-\mathrm{C} 8-\mathrm{H} 8 \mathrm{~B}$ & 109.2 \\
\hline $\mathrm{C} 3-\mathrm{C} 1-\mathrm{H} 1 \mathrm{~B}$ & 109.5 & $\mathrm{C} 9-\mathrm{C} 8-\mathrm{H} 8 \mathrm{~B}$ & 109.2 \\
\hline $\mathrm{H} 1 \mathrm{~A}-\mathrm{C} 1-\mathrm{H} 1 \mathrm{~B}$ & 109.5 & $\mathrm{H} 8 \mathrm{~A}-\mathrm{C} 8-\mathrm{H} 8 \mathrm{~B}$ & 107.9 \\
\hline $\mathrm{C} 3-\mathrm{C} 1-\mathrm{H} 1 \mathrm{C}$ & 109.5 & $\mathrm{C} 4-\mathrm{C} 9-\mathrm{C} 8$ & $114.0(2)$ \\
\hline $\mathrm{H} 1 \mathrm{~A}-\mathrm{C} 1-\mathrm{H} 1 \mathrm{C}$ & 109.5 & $\mathrm{C} 4-\mathrm{C} 9-\mathrm{H} 9 \mathrm{~A}$ & 108.7 \\
\hline $\mathrm{H} 1 \mathrm{~B}-\mathrm{C} 1-\mathrm{H} 1 \mathrm{C}$ & 109.5 & $\mathrm{C} 8-\mathrm{C} 9-\mathrm{H} 9 \mathrm{~A}$ & 108.7 \\
\hline $\mathrm{C} 3-\mathrm{C} 2-\mathrm{H} 2 \mathrm{~A}$ & 109.5 & $\mathrm{C} 4-\mathrm{C} 9-\mathrm{H} 9 \mathrm{~B}$ & 108.7 \\
\hline $\mathrm{C} 3-\mathrm{C} 2-\mathrm{H} 2 \mathrm{~B}$ & 109.5 & $\mathrm{C} 8-\mathrm{C} 9-\mathrm{H} 9 \mathrm{~B}$ & 108.7 \\
\hline $\mathrm{H} 2 \mathrm{~A}-\mathrm{C} 2-\mathrm{H} 2 \mathrm{~B}$ & 109.5 & $\mathrm{H} 9 \mathrm{~A}-\mathrm{C} 9-\mathrm{H} 9 \mathrm{~B}$ & 107.6 \\
\hline $\mathrm{C} 3-\mathrm{C} 2-\mathrm{H} 2 \mathrm{C}$ & 109.5 & $\mathrm{O}-\mathrm{C} 10-\mathrm{N}$ & $122.39(18)$ \\
\hline $\mathrm{H} 2 \mathrm{~A}-\mathrm{C} 2-\mathrm{H} 2 \mathrm{C}$ & 109.5 & $\mathrm{O}-\mathrm{C} 10-\mathrm{C} 7$ & $121.11(18)$ \\
\hline $\mathrm{H} 2 \mathrm{~B}-\mathrm{C} 2-\mathrm{H} 2 \mathrm{C}$ & 109.5 & $\mathrm{~N}-\mathrm{C} 10-\mathrm{C} 7$ & $116.49(16)$ \\
\hline $\mathrm{C} 1-\mathrm{C} 3-\mathrm{C} 2$ & $110.9(2)$ & $\mathrm{C} 16-\mathrm{C} 11-\mathrm{C} 12$ & $119.56(18)$ \\
\hline $\mathrm{C} 1-\mathrm{C} 3-\mathrm{C} 4$ & $114.6(2)$ & $\mathrm{C} 16-\mathrm{C} 11-\mathrm{N}$ & $122.18(18)$ \\
\hline $\mathrm{C} 2-\mathrm{C} 3-\mathrm{C} 4$ & $111.5(2)$ & $\mathrm{C} 12-\mathrm{C} 11-\mathrm{N}$ & $118.26(17)$ \\
\hline $\mathrm{C} 1-\mathrm{C} 3-\mathrm{H} 3 \mathrm{~A}$ & 106.5 & $\mathrm{C} 13-\mathrm{C} 12-\mathrm{C} 11$ & $120.2(2)$ \\
\hline $\mathrm{C} 2-\mathrm{C} 3-\mathrm{H} 3 \mathrm{~A}$ & 106.5 & $\mathrm{C} 13-\mathrm{C} 12-\mathrm{H} 12 \mathrm{~A}$ & 119.9 \\
\hline $\mathrm{C} 4-\mathrm{C} 3-\mathrm{H} 3 \mathrm{~A}$ & 106.5 & $\mathrm{C} 11-\mathrm{C} 12-\mathrm{H} 12 \mathrm{~A}$ & 119.9 \\
\hline $\mathrm{C} 5-\mathrm{C} 4-\mathrm{C} 9$ & $117.8(2)$ & $\mathrm{C} 14-\mathrm{C} 13-\mathrm{C} 12$ & $120.5(2)$ \\
\hline $\mathrm{C} 5-\mathrm{C} 4-\mathrm{C} 3$ & $125.0(2)$ & $\mathrm{C} 14-\mathrm{C} 13-\mathrm{H} 13 \mathrm{~A}$ & 119.7 \\
\hline $\mathrm{C} 9-\mathrm{C} 4-\mathrm{C} 3$ & $117.1(2)$ & $\mathrm{C} 12-\mathrm{C} 13-\mathrm{H} 13 \mathrm{~A}$ & 119.7 \\
\hline $\mathrm{C} 4-\mathrm{C} 5-\mathrm{C} 6$ & $121.3(2)$ & $\mathrm{C} 13-\mathrm{C} 14-\mathrm{C} 15$ & $119.2(2)$ \\
\hline $\mathrm{C} 4-\mathrm{C} 5-\mathrm{H} 5 \mathrm{~A}$ & 119.3 & $\mathrm{C} 13-\mathrm{C} 14-\mathrm{H} 14 \mathrm{~A}$ & 120.4 \\
\hline
\end{tabular}




$\begin{array}{llll}\mathrm{C} 6-\mathrm{C} 5-\mathrm{H} 5 \mathrm{~A} & 119.3 & \mathrm{C} 15-\mathrm{C} 14-\mathrm{H} 14 \mathrm{~A} & 120.4 \\ \mathrm{C} 7-\mathrm{C} 6-\mathrm{C} 5 & 120.8(2) & \mathrm{C} 16-\mathrm{C} 15-\mathrm{C} 14 & 120.9(2) \\ \mathrm{C} 7-\mathrm{C} 6-\mathrm{H} 6 \mathrm{~A} & 119.6 & \mathrm{C} 16-\mathrm{C} 15-\mathrm{H} 15 \mathrm{~A} & 119.6 \\ \mathrm{C} 5-\mathrm{C} 6-\mathrm{H} 6 \mathrm{~A} & 119.6 & \mathrm{C} 14-\mathrm{C} 15-\mathrm{H} 15 \mathrm{~A} & 119.6 \\ \mathrm{C} 6-\mathrm{C} 7-\mathrm{C} 10 & 123.12(19) & \mathrm{C} 15-\mathrm{C} 16-\mathrm{C} 11 & 119.6(2) \\ \mathrm{C} 6-\mathrm{C} 7-\mathrm{C} 8 & 118.97(19) & \mathrm{C} 15-\mathrm{C} 16-\mathrm{H} 16 \mathrm{~A} & 120.2 \\ \mathrm{C} 10-\mathrm{C} 7-\mathrm{C} 8 & 117.54(18) & \mathrm{C} 11-\mathrm{C} 16-\mathrm{H} 16 \mathrm{~A} & 120.2 \\ & & & \\ \mathrm{C} 1-\mathrm{C} 3-\mathrm{C} 4-\mathrm{C} 5 & 14.4(4) & \mathrm{C} 11-\mathrm{N}-\mathrm{C} 10-\mathrm{C} 7 & 175.31(18) \\ \mathrm{C} 2-\mathrm{C} 3-\mathrm{C} 4-\mathrm{C} 5 & -112.5(3) & \mathrm{C} 6-\mathrm{C} 7-\mathrm{C} 10-\mathrm{O} & -142.6(2) \\ \mathrm{C} 1-\mathrm{C} 3-\mathrm{C} 4-\mathrm{C} 9 & -170.4(3) & \mathrm{C} 8-\mathrm{C} 7-\mathrm{C} 10-\mathrm{O} & 30.4(3) \\ \mathrm{C} 2-\mathrm{C} 3-\mathrm{C} 4-\mathrm{C} 9 & 62.7(3) & \mathrm{C} 6-\mathrm{C} 7-\mathrm{C} 10-\mathrm{N} & 38.6(3) \\ \mathrm{C} 9-\mathrm{C} 4-\mathrm{C} 5-\mathrm{C} 6 & 2.7(3) & \mathrm{C} 8-\mathrm{C} 7-\mathrm{C} 10-\mathrm{N} & -148.4(2) \\ \mathrm{C} 3-\mathrm{C} 4-\mathrm{C} 5-\mathrm{C} 6 & 177.9(2) & \mathrm{C} 10-\mathrm{N}-\mathrm{C} 11-\mathrm{C} 16 & 41.7(3) \\ \mathrm{C} 4-\mathrm{C} 5-\mathrm{C} 6-\mathrm{C} 7 & 14.9(3) & \mathrm{C} 10-\mathrm{N}-\mathrm{C} 11-\mathrm{C} 12 & -138.7(2) \\ \mathrm{C} 5-\mathrm{C} 6-\mathrm{C} 7-\mathrm{C} 10 & 172.7(2) & \mathrm{C} 16-\mathrm{C} 11-\mathrm{C} 12-\mathrm{C} 13 & -1.9(3) \\ \mathrm{C} 5-\mathrm{C} 6-\mathrm{C} 7-\mathrm{C} 8 & -0.2(3) & \mathrm{N}-\mathrm{C} 11-\mathrm{C} 12-\mathrm{C} 13 & 178.47(19) \\ \mathrm{C} 6-\mathrm{C} 7-\mathrm{C} 8-\mathrm{C} 9 & -29.0(3) & \mathrm{C} 11-\mathrm{C} 12-\mathrm{C} 13-\mathrm{C} 14 & 0.5(3) \\ \mathrm{C} 10-\mathrm{C} 7-\mathrm{C} 8-\mathrm{C} 9 & 157.8(2) & \mathrm{C} 12-\mathrm{C} 13-\mathrm{C} 14-\mathrm{C} 15 & 1.2(4) \\ \mathrm{C} 5-\mathrm{C} 4-\mathrm{C} 9-\mathrm{C} 8 & -32.6(3) & \mathrm{C} 13-\mathrm{C} 14-\mathrm{C} 15-\mathrm{C} 16 & -1.6(4) \\ \mathrm{C} 3-\mathrm{C} 4-\mathrm{C} 9-\mathrm{C} 8 & 151.8(2) & \mathrm{C} 14-\mathrm{C} 15-\mathrm{C} 16-\mathrm{C} 11 & 0.2(4) \\ \mathrm{C} 7-\mathrm{C} 8-\mathrm{C} 9-\mathrm{C} 4 & 44.7(3) & \mathrm{C} 12-\mathrm{C} 11-\mathrm{C} 16-\mathrm{C} 15 & 1.5(3) \\ \mathrm{C} 11-\mathrm{N}-\mathrm{C} 10-\mathrm{O} & -3.5(3) & \mathrm{N}-\mathrm{C} 11-\mathrm{C} 16-\mathrm{C} 15 & -178.9(2) \\ & & & \end{array}$

Hydrogen-bond geometry $\left(\AA,{ }^{\circ}\right)$

\begin{tabular}{lllll}
\hline$D-\mathrm{H}^{\cdots} \cdots A$ & $D-\mathrm{H}$ & $\mathrm{H} \cdots A$ & $D \cdots A$ & $D-\mathrm{H}^{\cdots} \cdot A$ \\
\hline $\mathrm{N}-\mathrm{H} 0 A \cdots \mathrm{O}^{\mathrm{i}}$ & 0.86 & 2.27 & $3.054(2)$ & 151 \\
\hline
\end{tabular}

Symmetry code: (i) $x+1, y, z$. 